\title{
consolidation dynamique et construction en zones de décharge
}

\author{
par \\ M. Gambin \\ P. Guinnement \\ Techniques Louis Ménard, Paris, Granville
}

\begin{abstract}
RESUME Le budget consacré à 1 'amélioration des sols de fondation dans leur masse a été croissant ces dernières décennies. Les méthodes utilisées permettent en effet par un traitement modulé du sol selon ses variations, d'obtenir un terrain homogène. Cette solution qui permet tout ajustement ultérieur du plan masse est en effet plus souple qu'une solution sur pieux. Le champ d'application de ces méthodes s'est également élargi. Il englobe maintenant, grâce à la Consolidation Dynamique MENARD, les remblais rocheux et même des matériaux qui ne sont pas des sols mais qui le deviennent par destination : les remblais de déchets industriels et même d'ordures ménagères. Les auteurs montrent ici comment cette Consolidation Dynamique permet de transformer des matériaux de caractéristiques très varjables en sol porteur en décrivant un certain nombre de réalisations dont quelques-unes remontent à une dizaine d'années.
\end{abstract}

\section{INTRODUCTION}

A proximité des zones urbaines, nombreuses sont les dépressions naturelles ou artificielles (alors généralement d'anciennes exploitations de matériaux de construction) qui ont été comblées avec des remblais de natures diverses. Il s'agit généralement de matériaux de démolitions, de déblais de construction, de déchets industriels et d'ordures ménagères. Ces matériaux présen. tent les caractéristiques communes suivantes :

- grande compressibilité

- hétérogénéité importante

- potentiel de dégradation plus ou moins élevé.

Les problèmes posés par la réhabilitation de telles zones dans le cadre de l'amélioration de l'environnement, ont été résolus par la méthode de la Consolidation Dynamique MENARD qui évite la substitution de ces matériaux par d'autres plus nobles ou I'utilisation de fondations spéciales coûteuses comme préalable à tout aménagement.

Cette méthode permet de fonder superficiellement de nombreux types d'ouvrages : routes complexes sportifs, mais aussi petits immeubles d'habitation, hangars, ateliers, etc... Plus de 40 sites ont pu ainsi être rendus à l'urbanisation, tant en Europe que dans le nouveau monde.

\section{LA CONSOLIDATION DYNAMIQUE MENARD}

Il y a des siècles que l'on connait l'effet de compactage obtenu par l'application d'im- pacts à la surface du sol, mais il a fallu attendre les travaux de Louis MENARD, pour donner une autre dimension, inattendue, à cette méthode et en conséquence en voir les résultats décuplés et même multipliés par un facteur 100 (MENARD, 1974). Un autre article (LIAUSU, 1981) dans ces mêmes compterendus rappelie les bases de la méthode dont la complexité des mécanismes, variables selon les conditions locales, n'est pas toujours entièrement appréhendée (GAMBIN, 1979)

Nous insisterons simplement sur le fait qu'il s'agit d'un véritable compactage en masse rendant le sol homogène sur des épaisseurs atteignant souvent une dizaine de mètres (pour les cas qui nous occupent), à I'opposé de certaines méthodes de "vibrations et remplacement" qui conservent un caractère ponctuel (semblable aux pieux) et que cette technique s'applique ausi bien aux sols quasi-saturés qu'aux sols éloignés de la saturation, aux sols perméables comme aux sols imperméables.

Seules les déformations irréversibles ayant un effet durable de densification, l'action de la consolidation dynamique en profondeur s'explique plus par le rôle des contraintes de cisaillement que par celui des contraintes de compression (HARDIN, 1978).

On comprend ainsi mieux pourquoi la consolidation dynamique permet d'obtenir des résultats bien supérieurs à celui d'une surcharge qui sollicite le sol quasi-réversiblement.
La fig.1 rappelle les faits. 


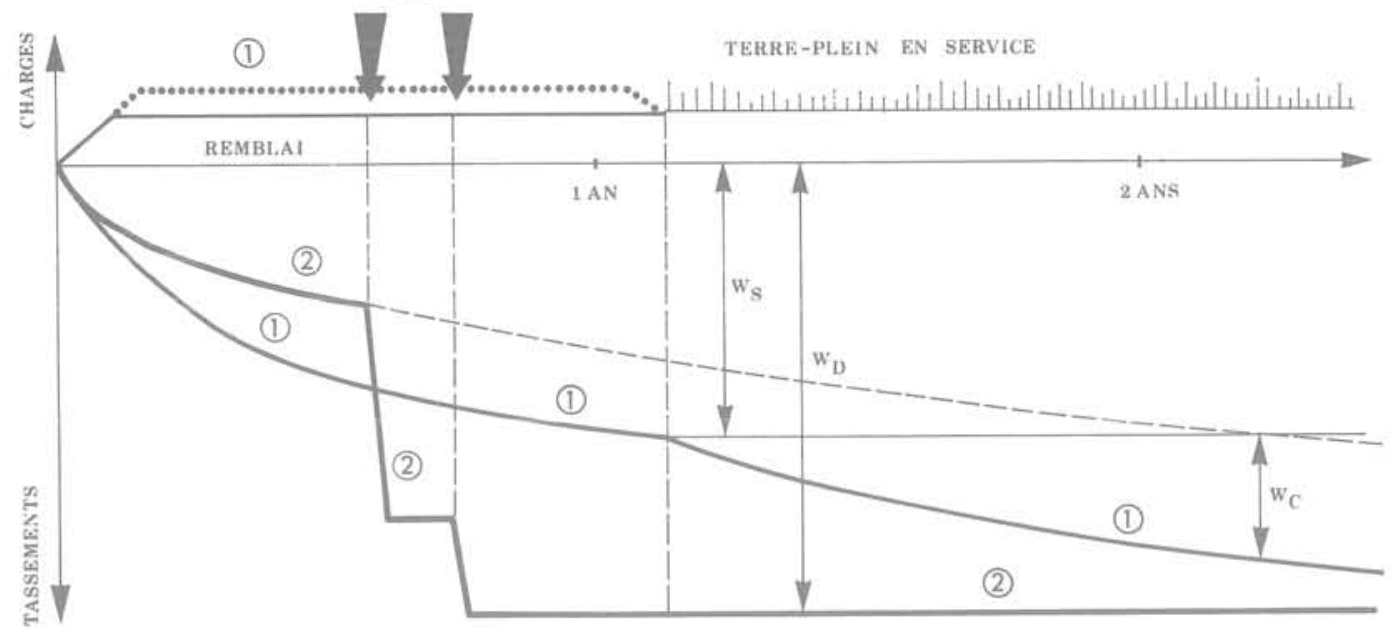

FIg. 1 COMPORTEMENT D'UN REMBLAI SUR SOL COMPRESSIBLE.

1) cas d'une surcharge.

2) Cas de la consolidation dynamique. $\mathrm{W}_{\mathrm{S}}=$ tassement du remblai "a stabilisation" par surcharge.

$\mathrm{W}_{\mathrm{C}}=$ tassement complémentaire du remblai dû à la mise en service. $\mathrm{W}_{\mathrm{D}}=$ tassement du remblai stabilisé par consolidation dynamique.

Enfin deux progrès importants dans le domaine de la géotechnique, ont permis de rendre la méthode fiable :

- les progrès dans la conception des capteurs placés au sein du sol.

- les progrès dans les essais in-situ.

En effet le compactage en masse ne peut se concevoir qu'accompagné de méthodes de suivi de chantier et de contrôle (contrôle entre passes et contrôle final) très strictes. Heureusement certains piézomètres à cellule fermée et les capteurs de pression totale type Geocell sont utilisables pendant les travaux. Enfin les essais in-situ, essentiellement les essais pressiométriques, sont devenus suffisamment courants et acceptés pour permettre une réception aisée du nouveau sol de fondation par le Mâtre d'Oeuvre.

3. INTERET DE LA CONSOLIDATION DYNAMIIQUE POUR LE TRAITEMENT DES DECHARGES.

Tout d'abord la méthode de consolidation dynamique est une méthode "passe-partout" applicable à tous les types de sol (MITCHELL 1979) depuis les enrochements (remblais avec blocs de plusieurs $\mathrm{m}^{3}$ ) jusqu'aux argiles (remblais de mottes d'argile aussi bien que limon argileux macroscopiquement homogène). On rappellera ainsi que les enrochements ne sont pas traités efficacement du tout par une surcharge (fig.1) et qu'ils sont contr'indiqués pour toute solution de pieux économique.

Ensuite la consolidation dynamique est une méthode de traitement global du sol, ce qui permet de livrer à l'aménageur un nouveau terrain avec des caractéristiques mécaniques uniformes et garanties.

Enfin en plus de cette augmentation considérable de la portance, concomittente avec une réduction notable des tassements totaux prévisibles à long terme et encore plus des tassements différentiels, la méthode permet de réduire-dans une certaine mesure- la vitesse de décomposition des matières organiques, oxydation et action des micro-organismes aérobies devenant plus difficiles.

\section{EXEMPLES D'APPLICATION DE LA METHODE}

Un certain nombre d'exemples de la méthode appliquée aux zones de décharge ont déjà été présentés : pour l'aire de fabrication des plate-formes d'exploitation du pétrole en mer, à Methyl, Ecosse (D'APPOLONIA, 1978), pour des entrepôts à Cwmbran, Pays de Galles (DOWNIE et TREHARE, 1979), pour la déviation de la voie ferrée Nüremberg-Bamberg (FLOSS 1980), pour l'échangeur d'une voie express à Redditch, Angleterre (CHARLES, 1981). D'autres exemples donneront lieu à des comptes-rendus ultérieurs, comme la nouvelle zone de stockage de produits pétroliers d'Amoco à Whiting, Indiana (E.U.), le tronçon de l'autoroute périphérique M25 à Waltham Cross près de Londres (G.B.), etc...

Nous décrivons ici 4 sites, plus particulièrement localisés dans l'ouest de la France:

Saint-1ô (Manche). Dans le but de fonder 9 pavillons à rez de chaussée, l'office Public d'H.L.M. de la Manche avait retenu la Consolidation Dynamique pour traiter, en ville, une ancienne dépression, remblayée immédiatement après la guerre de matériaux de démolitions et débris divers sur $4 \mathrm{~m}$ d'épaisseur. Un tassement de 15 à $20 \mathrm{~cm}$ a été obtenu pour une énergie de 140 à $200 \mathrm{t} \times \mathrm{m}$ $/ \mathrm{m}^{2}$. Comme le montre la fig.2 la portance du terrain a été améliorée par un facteur de 3 à 4 et le taux de travail admissible après traitement était surabondant. Les immeubles terminés en 1973, n'ont subi aucune déforma- 


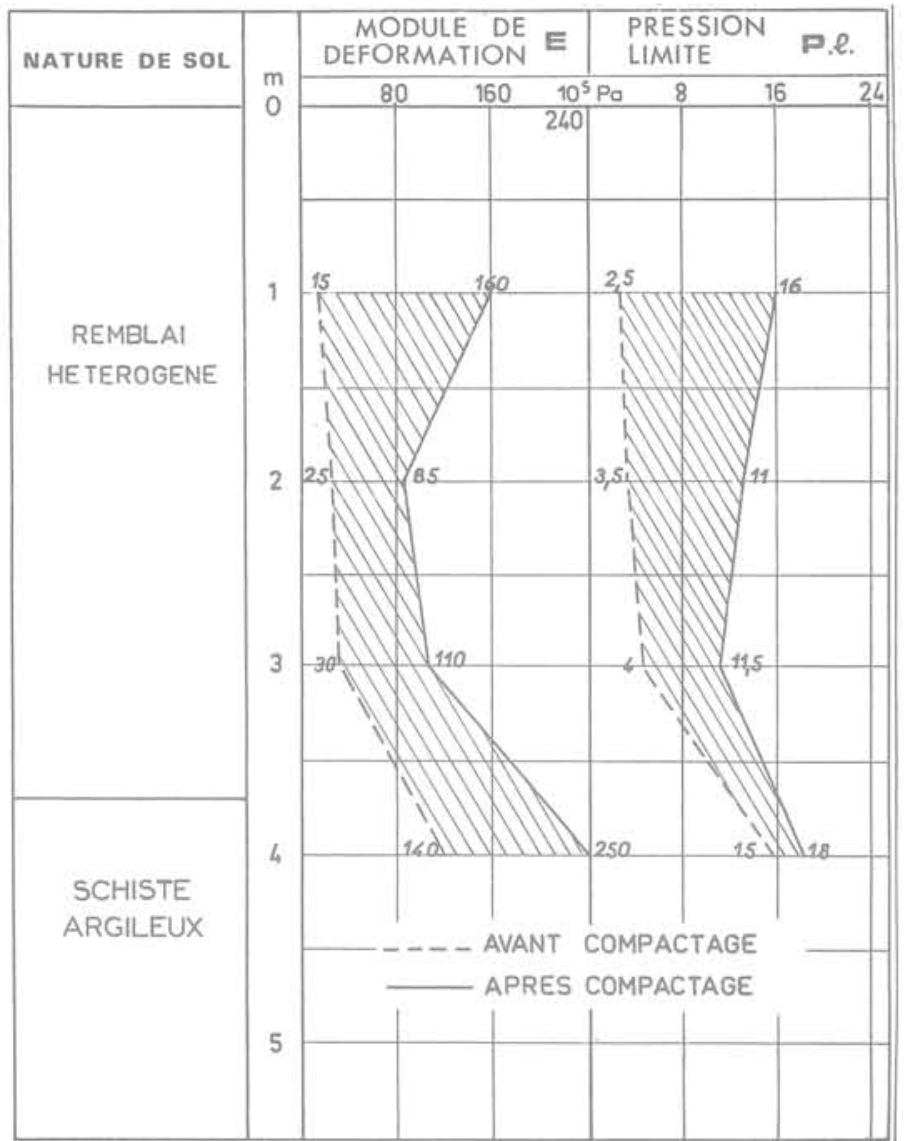

FIg.2 AMELIORATION DES CARACTERISTIQUES MECANIQUES (MESUREES AU PRESSIOMETRE) à SAINT-LO.

tion $($ fig. 3$)$.

Coutances (Manche). La fondation d'un dallage chargé à $30 \mathrm{KPa}$ et de la couverture des magasins et ateliers Casam a pu être assurée en surface sur un dépôt ancien d'ordures ménagères et de limon de 7 à $10 \mathrm{~m}$ d'épaisseur. Le traitement a entraîné un abais-

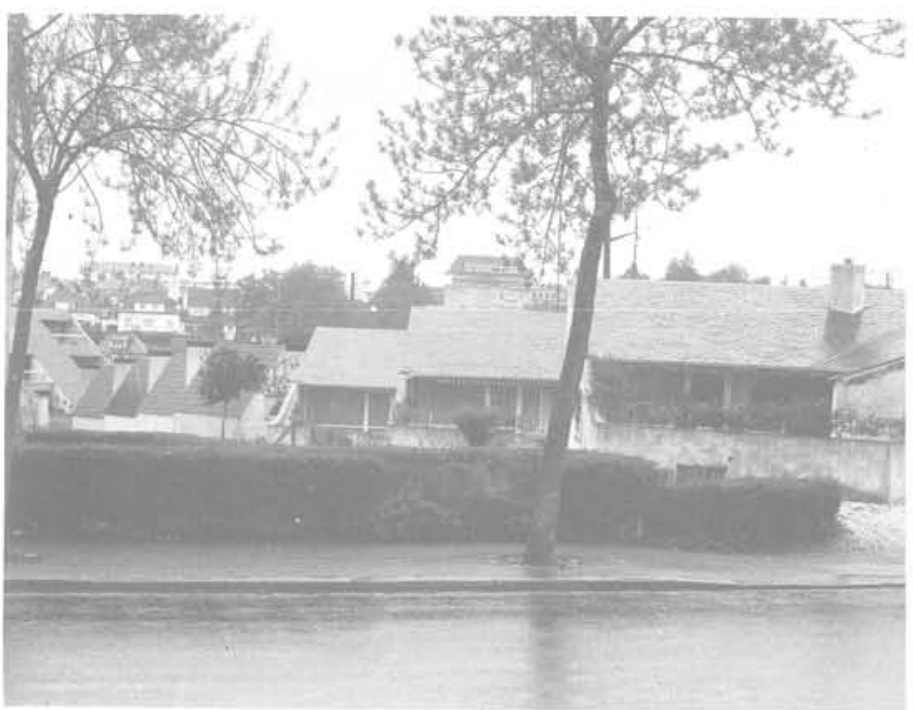

Fig. 3 ASPECT DES PAVILLONS DE SAINT-LO EN 1977 .

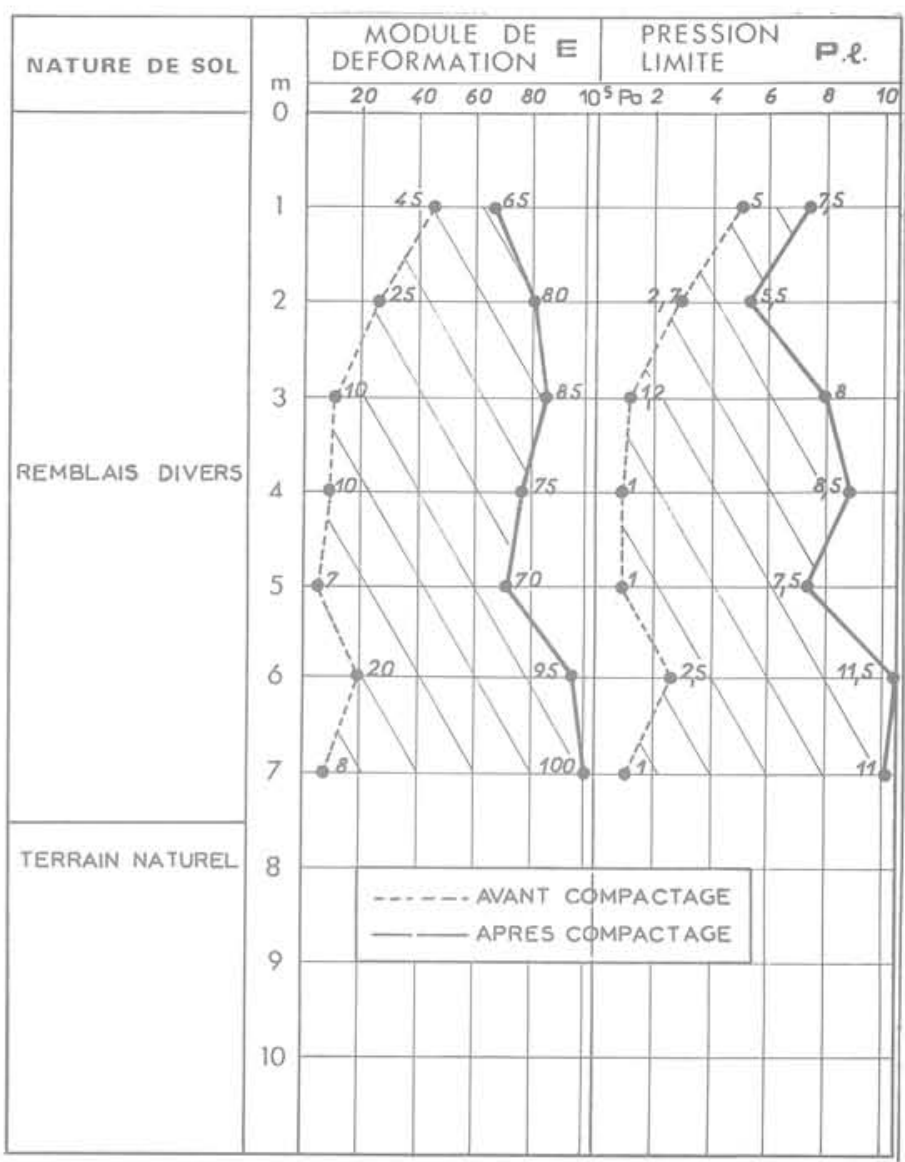

FIg. 4 AMELIORATION DES CARACTERISTIQUES MECANIQUES (MESUREES AU PRESSIOMETRE) à COUTANCES.

sement du niveau du sol de 1,75 m en moyenne poyr une énergie en 2 passes de $250 t$ $\mathrm{x} \mathrm{m} / \mathrm{m}^{2}$ et a plus que triplé les caractéristiques mécaniques (fig.4). Un taux de travail de $200 \mathrm{KPa}$ a pu être recommandé et depuis 1973 aucun tassement n'a été observé dans le dallage ni la structure (fig.5).

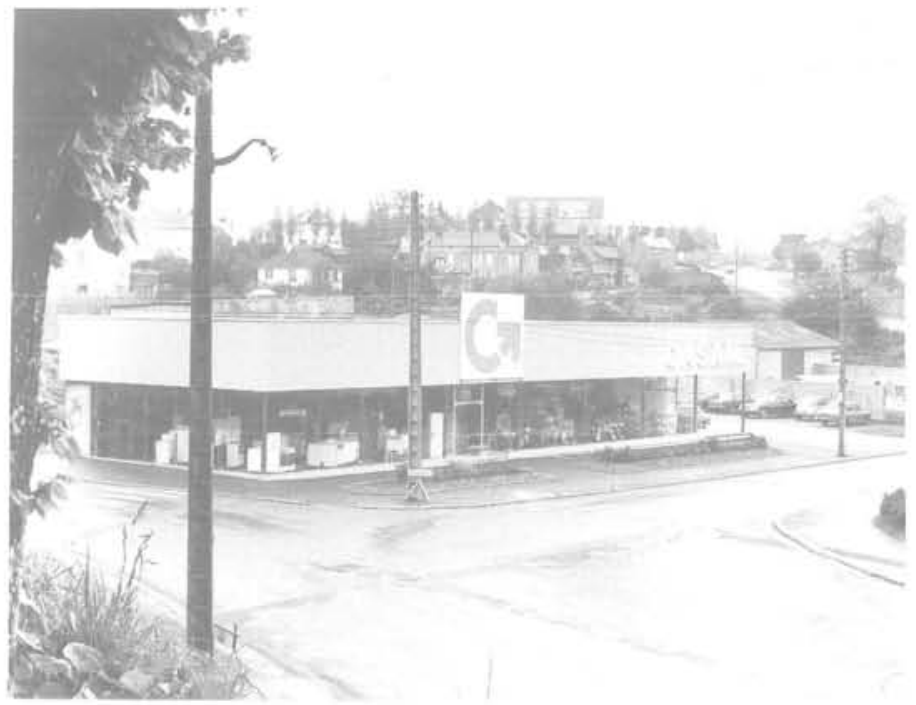

Fig.5 VUE D'ENSEMBLE DU MAGASIN DE COUTANCES EN 1977. 
Petit Quevilly (Seine-Maritime). La Société d'Aménagement de là Région de Rouen a retenu en 1972 le principe de la consolidation dynamique dans le but de traiter un remblai de déchets industriels sur 9 à $10 \mathrm{~m}$ d'épaisseur de manière à stabiliser les VRD d'une future zone industrielle. Le traitement a abaissé le niveau du sol de 40 à 80 $\mathrm{cm}$, pour ung énergie moyenne en 2 passes de $300 \mathrm{t} \times \mathrm{m} / \mathrm{m}^{2}$, les zones où ce tassement provoqué était supérieur à $1 \mathrm{~m}$ ont dû être partiellement purgées de poches de sciures et de graisses incompressibles. Depuis leur réalisation, les voieries et réseaux n'ont manifesté aucune déformation ( $\mathrm{fig} \cdot 6)$.

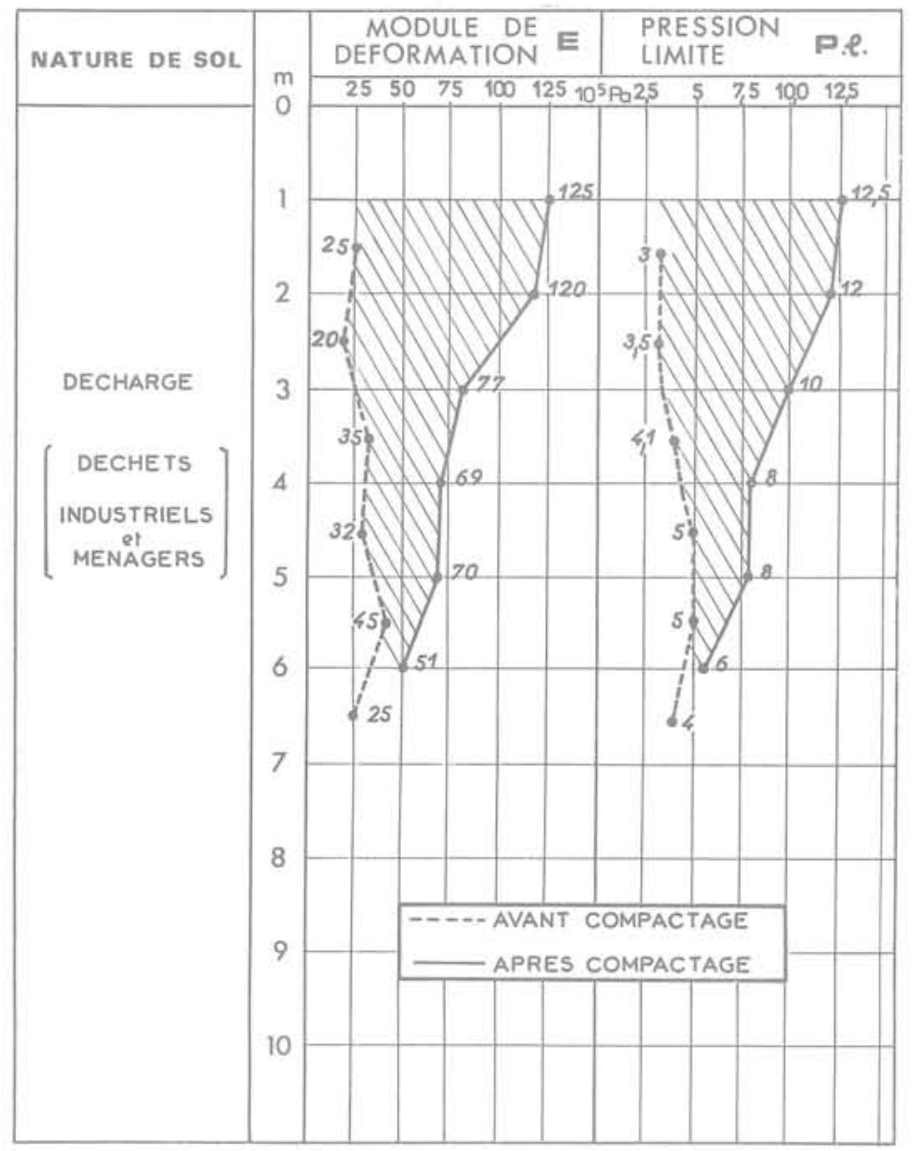

FIg. 6 AMELIORATION DES CARACTERISTIQUES MECANIQUES (MESUREES AU PRESSIOMETRE) à PETIT QUEVILLY.

Saint-Nazaire (Loire Atlantique) : la voie express dite pénétrante Ouest traverse un dépôt d'ordures entre l'échangeur de Certé et le pont de la Missaudière. Cette ancienne décharge municipale, d'un volume de $30000 \mathrm{~m}^{3}$, constituée entr'autres de carcasses de voitures reposait sur une argile plastique. D'abord régalées sur la totalité de l'emprise du remblai d'accès au pont, les ordures, alors d'une épaisseur de $1,5 \mathrm{~m}$ seulement furent recouvertes d'au moins $1 \mathrm{~m}$ de matériaux sableux. Le but du traitement était le compactage en masse de ces 2 matériaux sans toutefois remanier 1'argile sousjacente. Les essais pressiométriques réalisés ultérieurement ont montré qu'en fait, malgré l'énergie réduite apnliquée par coun ( 8 tonnes tombant de $5 \mathrm{~m}$ ) les 3 premiers mètres d'argile ont bénéficié d'une amélioration de 30 à $50 \%$ de leurs caractéristiques mécaniques. Le tassement moyen a été de 1 'ordre de $12 \%$ de la hauteur totale du remblai traitá, les valeurs les plus élevées étant relevées là où la décharge était la moins haute initialement.

Le chantier avait été suivi très soigneusement par le CETE d'Angers qui avait mis en place 10 cellules de tassement et 4 sondes de mesures de pression interstitielles dans l'argile sous-jacente. Les observations ont confirmé qu'il était possible de limiter I'action sur l'argile pendant la consolidation dynamique des remblais.

\section{PERSPECTIVES D'AVENIR}

Les recherches en cours sur la métrode de la consolidation dynamique concernent de nombreux domaines à des niveaux variés = recherche fondamentale sur le comportement des sols naturels fins saturés, rôle des contraintes de cisaillement, rôle de la liquéfaction, rôle du regain thixotropique, etc. mais aussi recherche appliguée sur la profondeur d'action en fonction des différents paramètres de la méthode (poids et surface du pilon, hauteur de chute, type de matériau), sur le comportement ultérjeur du matériau, essentiellement son tassement à long terme, sur la propagation des vibrations au voisinage, etc...

Si un certain nombre de règles ont déjà pu être données dans le domaine de l'application à des matériaux homogènes, même des remblais d'argile, leur extrapolation aux remblais d'ordures ménagères ou de déchets industriels n'est pas toujours aisée.

Chaque chantier de ce type est un cas particulier, qui nécessite une investigation spéciale. Toutefois, on est assuré gue Ia méthode est applicable et qu'elle donnera de bons résultats, à l'excention du cas des ordures ménagères récentes entièrement noyées, et à condition de pouvoir mettre en jeu des énergies plus importantes que pour un sol plus homogène. On attirera l'attention sur la difficulté de réaliser une zone d'essai valable; en effet, souvent ces zones ont une surface trop réduite, comptetenu de la règle qqu'une bande pérịhériquo de largeur égale à la profondeur dì terrain à compacter doit être traitée en plus de 1 a surface utilisable. C'est la raison majeure des mécomptes qui ont pu être rencontrés antérieurement, par exemple lors de l'essai de Corby (CHARLES, 1978). Bien évidemment les techniques de contrôle de l'efficacité de la méthode pendant son application sont plus délicates, toutefois l'expérience montre que dans la majorité des cas, il est possible de mener à bien des essais in-situ significatifs.

\section{CONCLUSION}

Les quelques exemples donnés ci-dessus ont montré l'intérêt indéniable de la Consolida- 
tion Dynamique pour le traitement des zones de décharges ménagères ou industrielles et la transformation d'un matériau de rebut en un sol de fondation. Si les paramètres profondeur et hétérogénéité ne semblent pas limiter l'efficacité de la méthode, par contre la présence d'une nappe phréatique à faible profondeur peut être un élément défavorable. Habituellement les énergies à mettre en jeu sont plus importantes que pour un sol naturel à épaisseur comparable, mais cette règle n'est pas générale.

Une grande avenue reste ouverte à la recherche dans ce domaine, mais l'extrapolation des résultats obtenus sur des sols relativement homogènes à de tels matériaux restera toujours aléatoire. Seuls la zone d'essai de taille_convenable et le recours aux expériences antérieures en vraie grandeur permettent au spécialiste d'engager sa responsabilité quant à la bonne fin d'un traitement.

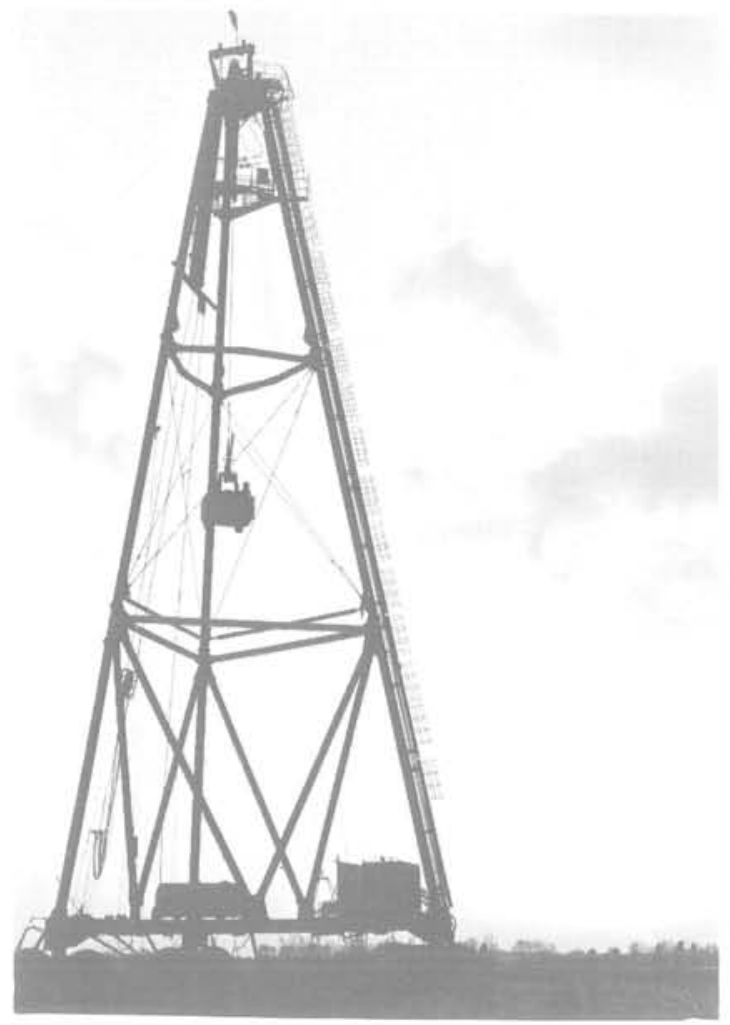

Fig.7 EQUIPEMENT MENARD POUR TRAITEMENT DE PLUS DE $10 \mathrm{M}$ D'EPAISSEUR DE SOL.

(photo prise en Afrique du Sud).
D'APPOLONIA D. (1978) Foundation Improvement by Dynamic Consolidation, ASCE -N.Y. Section, Foundation and Soil Mechanics Group Seminar on Improving Poor Soil Conditions (26 oct.).

CHARLES J.A., BURFORD D., WATTS K.S. (1981) Field Studies of the Effectiveness of the Ground Treatment Technique "Dynamic Consolidation" 10th ICSMFE Stockholm (June).

CHARLES J.A., EARLE E.W., BURFORD D. (1978) Treatment and subsequent performance of cohesive fill left by opencast ironstone mining at Snatchill experimental housing site Corby, Conference on Clay Fills, ICE London.

DOWNIE A.R. and TREHARE G. (1979) Dynamic Consolidation of Refuse at Cwbran, Symposium on the Engineering Behaviour of Industrial and Urban Fill, Birmingham University (Apri1).

FLOSS R. (1980) Consolidation Dynamique de terrains compressibles pour fondation de remblais autoroutiers et ferroviaires, Colloque International du Compactage, ENPC Paris (Avril).

GAMBIN M. (1979) Menard Dynamic Consolidation, ASCE-Washington DC Section, Seminar on Ground Reinforcement (Janvier), aussi Sols Soils $n^{\circ} 29$.

HARDIN B.0. (1978) The Nature of Stress Strain Behaviour for Soils, State of the Art Report, ASCE Specialty Conference on Earthquake Engineering and Soil Dynamics Pasadena, Californie (Juin).

LIAUSU P. (1981) Etude des Vibrations engendrées par le compactage dynamique, 2èmes Jounées Nationales Géotechniques, Nantes (Avril).

MENARD L. (1974). Un procédé de consolidation $=1 \mathrm{e}$ pilonnage intensif, Supplément aux aux Annales de I'ITBTP (Sept.).

MITCHELL J.K. (1979). Ground Reinforcement Techniques, an overwiew, ASCE-Washington DC Section, Seminar on Ground Reinforcement ( J anuary). 